\title{
Reconstrucciones virtuales en 3D del repertorio cerámico de cazadores- recolectores de la costa nordeste del Chubut, Patagonia argentina
}

\section{( Verónica Schuster** y Mirsha Quinto Sánchez **}

\section{Resumen}

El objetivo de este trabajo es presentar la aplicación de un método hasta el momento nunca empleado en el estudio de la cerámica de grupos cazadores-recolectores de la Patagonia argentina: la reconstrucción virtual en tres dimensiones (3D) a partir de piezas parcialmente remontadas. En los contextos arqueológicos de la costa nordeste de la provincia del Chubut el material cerámico se presenta altamente fragmentado y si bien se han remontado o ensamblado parcialmente algunas piezas, hasta la aplicación de este método no se había obtenido la representación completa de las distintas morfologías presentes. Al respecto, además de desarrollar el procedimiento técnicometodológico mediante el cual se obtuvieron las imágenes gráficas virtuales en $3 \mathrm{D}$, también se presentará la información que de ello deriva, entre lo más destacable, la capacidad o el volumen y el peso de las piezas cerámicas. Dichas variables permiten, al menos inicialmente, plantear algunos aspectos relativos al uso y transporte de estos contenedores.

\section{D reconstructions of the ceramic assemblages of hunter-gatherers from the Northeastern coast of Chubut, Argentine Patagonia}

\begin{abstract}
The main aim of this article is to serve as an introduction to the use of a method not previously employed in the study of the pottery of hunter-gatherer groups from Patagonian Argentina: the three-dimensional (3D) virtual reconstruction of partially assembled containers. In the archaeological contexts of the study area - northeastern coast of the Chubut Province - the ceramic material is highly fragmented, and although some potsherds were partially assembled, complete representations of the different morphologies were not documented prior to the application of this method. In this
\end{abstract}

Recibido:

3 de marzo de 2018

Aceptado:

21 de junio de 2018

\section{Palabras clave}

Cerámica Reconstrucción $3 D$ Cazadores-recolectores Patagonia Argentina

Keywords

Pottery

3 D reconstruction Hunter-gatherers Argentine Patagonia 
regard, our paper shows the technical-methodological procedure by which the virtual graphic images were obtained in 3D. Furthermore, we also discuss the data resulting from this analysis, information relating to the volume - or capacity - and weight of the ceramic pieces. As an initial interpretative tool, these variables allow us to propose concepts related to the use and transportation of these containers.

\section{Introducción}

En la costa nordeste de la provincia del Chubut, los sitios arqueológicos en los que se ha recuperado cerámica son en su mayoría de superficie y el material es fragmentario casi en su totalidad (Schuster, 2014). Desde el año 2006 han estado aplicándose diversos tipos de análisis para explorar la variabilidad y la funcionalidad de esta tecnología (Gómez Otero, Schuster y Banegas, 2017a; Schuster, 2012). A partir de los análisis de las pastas y el estudio de la morfología y de la decoración fue posible vincular algunas piezas con otros lugares probables de procedencia como el oeste de Patagonia y/o centro-sur de Chile, si bien mayormente su manufactura es local (Gómez Otero et al., 2017a; Schuster, 2009, 2014, 2015; Schuster y Banegas, 2010; Schuster, Banegas y Taylor, 2013). Además, se ha explorado la función y el uso a partir de isótopos estables $\left(\mathrm{d}^{13} \mathrm{C}\right.$ y d $\left.{ }^{15} \mathrm{~N}\right)$ y cromatografía gaseosa, cuyos resultados revelaron que habrían sido utilizadas para preparar y cocinar alimentos, especialmente plantas y en menor medida, mamíferos terrestres y peces marinos (Gómez Otero, Schuster y Constenla, 2014; Gómez Otero, Schuster y Svoboda, 2014; Schuster, 2014). En esta oportunidad se presentan los primeros resultados obtenidos de la aplicación de la reconstrucción en 3D de las piezas parcialmente remontadas.

El empleo de modelos 3D no es un tema novedoso para la arqueología (Irujo Ruiz y Prieto Martínez, 2005). En el país ha sido implementado para estudios arqueológicos e históricos tendientes a la conservación (Ávido y Vitores, 2016; Ghiani Echenique, Sokol, y Lozano, 2017), no obstante lo cual hay pocos ejemplos de su aplicación al estudio cerámico (Carosio, Aguilar y Bárcena, 2013; Ghiani Echenique y León, 2013) y particularmente no se registran antecedentes para la región patagónica. Esta herramienta de reconstrucción digital resulta muy ventajosa en contextos arqueológicos en los que el material cerámico es fragmentario, existen bajas posibilidades de remontajes o ensamblajes y las piezas completas no se registran o son muy escasas, tal como ocurre en el caso de estudio que se presenta en este trabajo. El valor de la reconstrucción 3D se manifiesta no solo en la generación virtual de la morfología completa de los contenedores, sino también en la obtención de nuevos datos analíticos como el volumen o el peso de las piezas, los cuales permiten evaluar aspectos relacionados con el uso y la probable transportabilidad de estos contenedores cerámicos en el seno de grupos cazadores-recolectores móviles.

\section{Área de estudio, sitios arqueológicos y materiales cerámicos}

La costa noreste del Chubut se delimita por la desembocadura del Arroyo Verde al norte y el estuario del río Chubut al sur (Figura 1). Esta región, de clima árido y vegetación arbustiva y esteparia, se caracteriza por la abundante oferta de alimentos marinos y terrestres, aunque son limitadas las fuentes de agua dulce permanentes, siendo las únicas el río Chubut y los manantiales de tres bajos sin salida en Península Valdés (Figura 1).

El material cerámico se recuperó en bases residenciales y en menor frecuencia, en campamentos transitorios y localizaciones (Schuster, 2014). Los sitios son de superficie, se emplazan en ambientes de dunas y por ello usualmente se presentan sin asociación directa con restos orgánicos factibles de datar. En consecuencia, las inferencias 


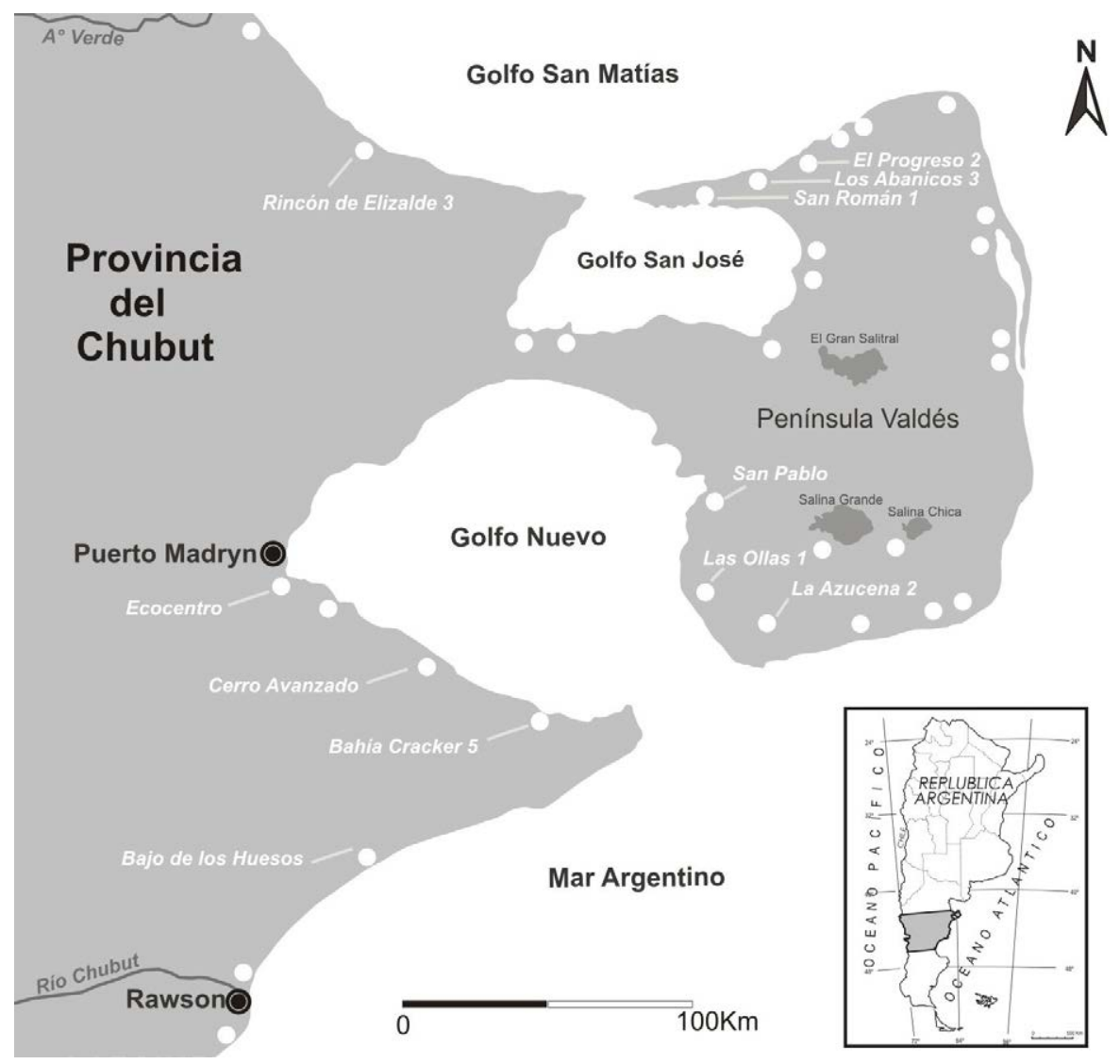

Figura 1. Área de estudio y sitos arqueológicos mencionados en este trabajo (Schuster, 2012, 2014).

cronológicas sobre el registro cerámico son aún limitadas en el área: $840 \pm 130{ }^{14} \mathrm{C}$ años AP (corrección por $\delta^{13} \mathrm{C}$ : $-20,1 \%$; sin corrección para el Efecto Reservorio) obtenido de valvas de un fogón del sitio Ecocentro en el golfo Nuevo sur; $980 \pm 70$ y $400 \pm 50$ ${ }^{14} \mathrm{C}$ años AP (carbón vegetal, sin calibrar) en el sitio San Pablo 7 (costa del golfo Nuevo en Península Valdés) (Gómez Otero et al., 2017a; Schuster, 2012, 2014; Figura 1). A partir de solo dos sitios datados no es posible evaluar la variabilidad temporal de esta tecnología en el área, aunque otras dataciones obtenidas para la provincia del Chubut permiten estimar su adopción e implementación para el Holoceno tardío (Aldazabal y Eugenio, 2009; Arrigoni, 2002; Caviglia, Borrero, Casiraghi, García y Horwitz, 1982; Gradin, 1980; Schuster, 2014; entre otros).

Se han registrado más de 1.300 fragmentos en 40 sitios arqueológicos (Gómez Otero et al., 2017a; Schuster, 2012, 2014; Figura 1). Usualmente, el material se presenta fragmentado, evidenciando erosión y desgaste. A pesar de estas características, a través de las tareas de ensamblaje se logró reconstruir parcialmente la morfología de 12 contenedores (Schuster, 2014). Para este trabajo se consideraron estas piezas reconstruidas y la única completa que se cuenta a la fecha para el área (Gómez Otero et al., 2017b) (Figura 2 y Tabla 1).

\section{Método}

El protocolo para confeccionar y obtener una reconstrucción 3D a partir de fragmentos cerámicos remontados se basa, siguiendo el trabajo realizado por Irujo Ruiz y Prieto Martínez (2005), en tres instancias que se mencionan a continuación. 


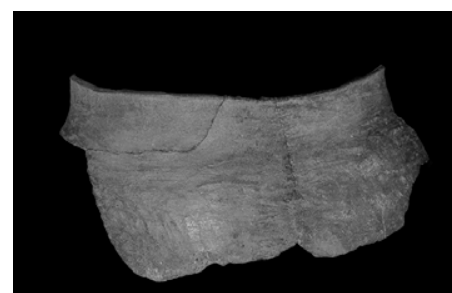

Los abanicos $3(2)$

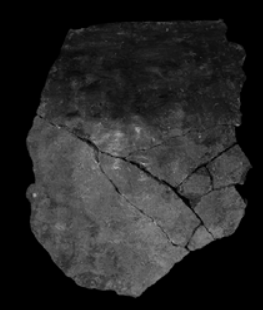

El Progreso 2(1)

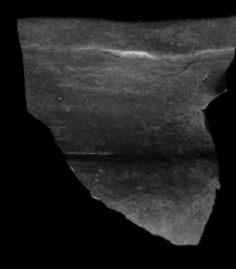

El Progreso 2(14)

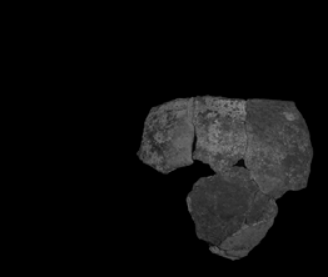

Rincon Elizalde 3(3)

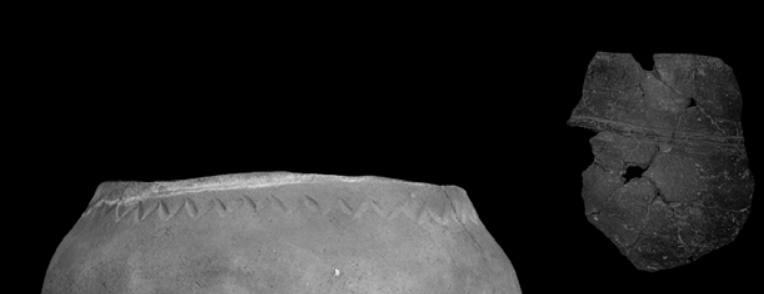

Cerro Avanzado

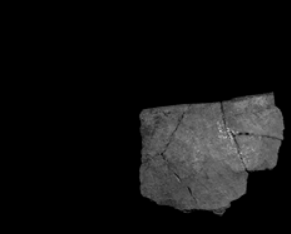

ElProgreso 2(7)

San Pablo

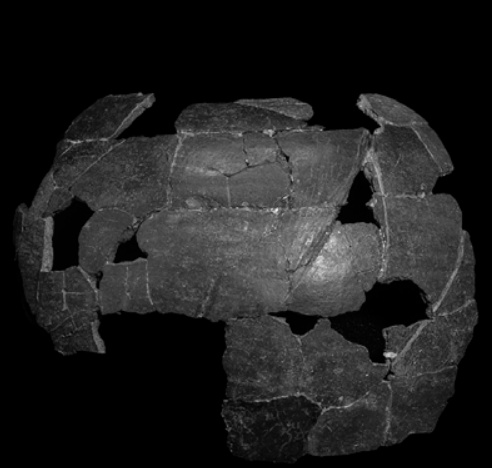

LaAzucena 2(1)

Figura 2. Algunas de las piezas cerámicas parcialmente remontadas y la única completa del área de estudio.

1. Generación de un dibujo del perfil 2D. Para realizar el perfil en 2D en un programa de diseño gráfico (InkDesing) es imprescindible contar con los dibujos y/o las fotografías laterales de los ensamblajes más completos, además del valor del diámetro de boca sensu Rice (1987). Comenzando de este diseño se obtiene un gráfico vectorial re-escalable (SVG o Scalable Vector Graphics) a partir de software libre (Inkscape, Librecad, Qcad) o cerrado (Adobe Illustrator, AutoCad) (Figura 3). Para el presente trabajo se utilizó Adobe Illustrator CC (17.1) y una cámara Nikon D300 con un lente $50 \mathrm{~mm}$ fijo 2.8, no obstante es posible trabajar con cualquier cámara con una resolución mayor a 6MP.

2. Elaboración del modelo 3D. Se efectúa a partir de la imagen $2 \mathrm{D}$ y por medio de un sólido o volumen de revolución que rotará alrededor de una recta o eje ubicado en el diseño SVG (Sopena Vicién, 2006), plano denominado como eje de rotación. Este proceso se realiza en alguna plataforma de reconstrucción o 


\begin{tabular}{|c|c|c|c|c|c|c|c|c|c|c|c|c|c|}
\hline 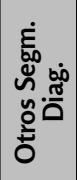 & 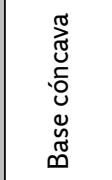 & ' & & ' & 荌 & 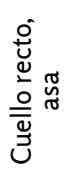 & 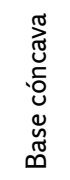 & ' & $\stackrel{\pi}{<}$ & ' & & ' & ' \\
\hline $\begin{array}{l}\frac{\pi}{60} \\
\frac{0}{0} \\
\frac{0}{0} \\
\Sigma \\
\Sigma\end{array}$ & 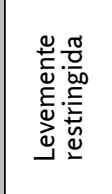 & 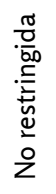 & 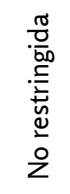 & 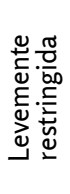 & 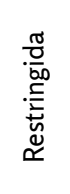 & 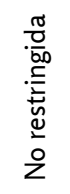 & 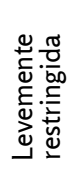 & 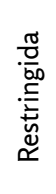 & 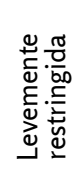 & 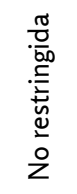 & 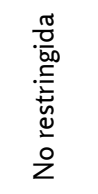 & 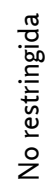 & 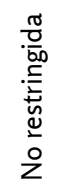 \\
\hline 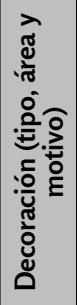 & 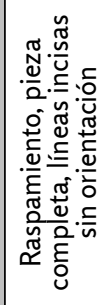 & ' & 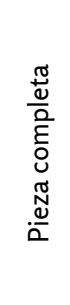 & ' & ' & ' & 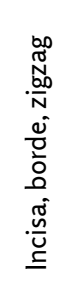 & ' & & 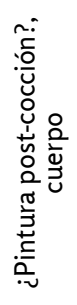 & 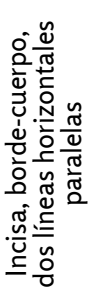 & & ' \\
\hline 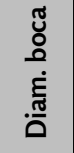 & $\stackrel{1}{\circ}$ & $\underset{\sim}{\infty}$ & $\stackrel{\sim}{N}$ & $\begin{array}{l}\text { II } \\
\text { İ }\end{array}$ & $\stackrel{్}{\circ}$ & 우 & ন & $\mathbb{J}$ & $\begin{array}{l}\text { I' } \\
\text { d̦ }\end{array}$ & 어 & $\stackrel{\circ}{-}$ & $l_{n}^{\prime}$ & $\approx$ \\
\hline 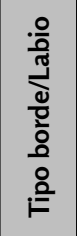 & 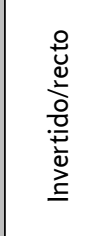 & 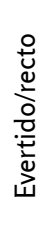 & 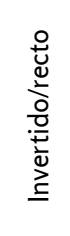 & 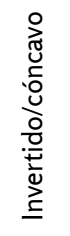 & 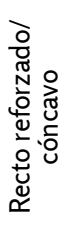 & 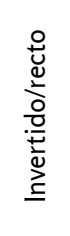 & 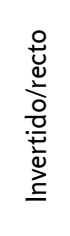 & 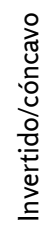 & 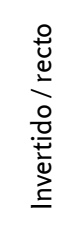 & 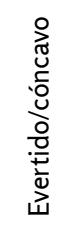 & 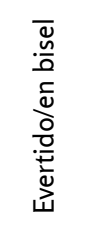 & 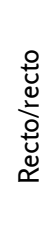 & 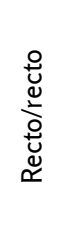 \\
\hline 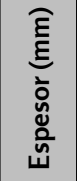 & 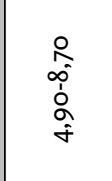 & 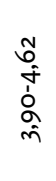 & $\begin{array}{l}\text { 早 } \\
0 \\
0 \\
0 \\
0 \\
\end{array}$ & 孜 & 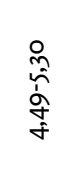 & $\stackrel{\infty}{m}$ & $\stackrel{+}{+}$ & 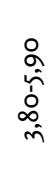 & $\begin{array}{l}\text { Oे } \\
\hat{\tilde{n}} \\
0 \\
\infty \\
n\end{array}$ & 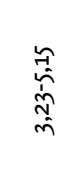 & 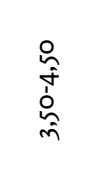 & $\stackrel{\infty}{n}$ & $\stackrel{m}{n}$ \\
\hline 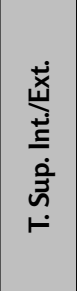 & 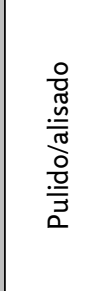 & $\begin{array}{l}\frac{}{\frac{0}{\bar{y}}} \\
\frac{2}{2} \\
\frac{0}{\overline{0}} \\
\frac{0}{2}\end{array}$ & 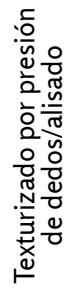 & 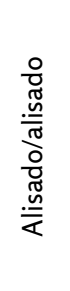 & $\begin{array}{l}\frac{}{\frac{0}{3}} \\
\frac{2}{2} \\
\frac{0}{0} \\
\frac{0}{3} \overline{2}\end{array}$ & 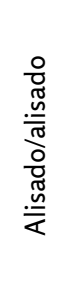 & 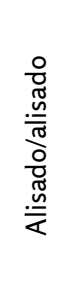 & $\begin{array}{l}\frac{0}{\frac{0}{3}} \\
\frac{2}{2} \\
\frac{0}{\frac{0}{5}} \\
\frac{0}{2}\end{array}$ & $\begin{array}{l}\frac{0}{\frac{0}{3}} \\
\frac{2}{2} \\
\frac{0}{\frac{0}{3}} \\
\frac{0}{3}\end{array}$ & 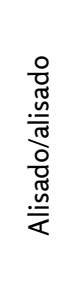 & $\begin{array}{l}\frac{0}{\pi} \\
\frac{.0}{\bar{\pi}} \\
\frac{\pi}{0} \\
\frac{0}{\overline{3}} \\
\frac{0}{3}\end{array}$ & 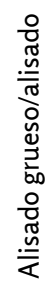 & 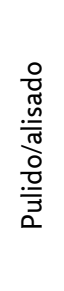 \\
\hline$\frac{\mathbb{d}}{2}$ & 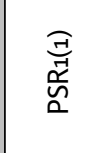 & 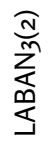 & $\begin{array}{l}\text { 곤 } \\
\text { Iิ }\end{array}$ & $\begin{array}{l}\stackrel{\bar{N}}{N} \\
\text { N }\end{array}$ & $\begin{array}{l}\stackrel{\mathcal{J}}{N} \\
\text { Ù }\end{array}$ & $\begin{array}{l}\widehat{\mathbb{J}} \\
\stackrel{\sigma}{\sigma}\end{array}$ & $\overline{\bar{n}}$ & $\stackrel{\sqrt{J}}{\frac{N}{S}}$ & 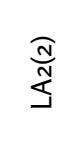 & 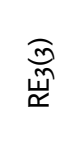 & 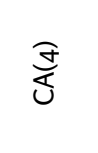 & 窇 & 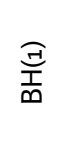 \\
\hline$\stackrel{\mathscr{n}}{n}$ & 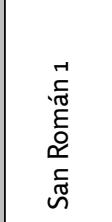 & 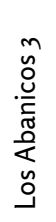 & 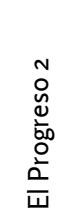 & 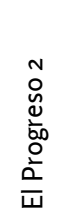 & 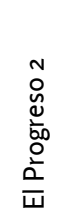 & $\begin{array}{l}\vec{y} \\
\overrightarrow{\tilde{\sigma}} \\
\overline{\overline{0}} \\
\vec{y} \\
\vec{J}\end{array}$ & 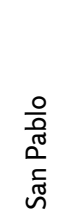 & 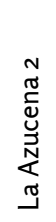 & 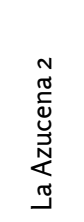 & 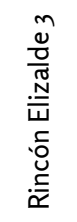 & 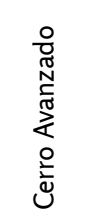 & 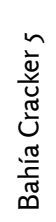 & 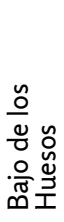 \\
\hline
\end{tabular}

Tabla 1. Características macroscópicas de las piezas reconstruidas en $3 D$.

Referencias. T. Sup. Ext./Int.: tratamiento de superficie externo e interno; Diam. Boca: diámetro de la boca; Otros Segm. Diag.: otros segmentos diagnósticos. 


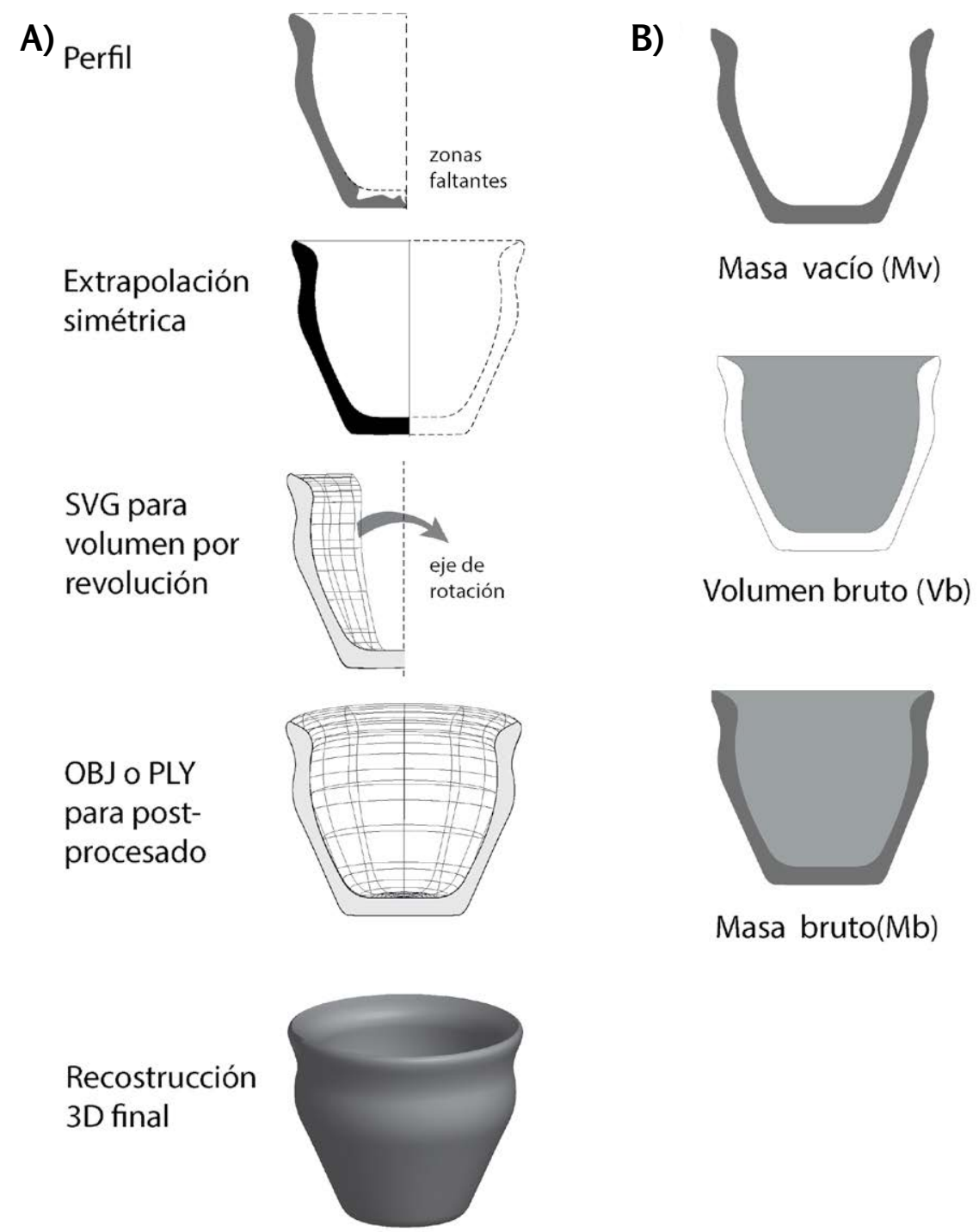

Figura 3. A) Esquematización del trabajo desarrollado para obtener las reconstrucciones $3 D$ y $B$ ) representación del volumen bruto $(\mathrm{Vb})$, masa vacío $(\mathrm{Mv})$ y masa bruto $(\mathrm{Mb})$ según la propuesta de Sopena Vicién (2006).

animación 3D que pueda realizar un volumen por revolución (Strata Desing 3D, Blender, Autocad, OpenSCAD, CAD3D, FreeCAD, BRL-CAD). En el caso del presente artículo se empleó Strata Desing 3D CX 5.1. Los decorados no fueron tomados para análisis ya que la reconstrucción por revolución extrapolaría también cualquier decoración del perfil, alterando los cálculos de volumen del mismo. Una vez realizado el proceso, el sólido o volumen se exporta como un archivo formato OBJ (que contiene la información de la geometría 3D incluyendo la posición de cada vértice) o PLY (archivo de lista de polígonos contenidos en nuestra reconstrucción) (Figura 3).

3. Post-procesado de las imágenes obtenidas. Este paso permite establecer el acabado, la textura y el color que presentarán las piezas reconstruidas en 3D y se realiza en un programa para reconstrucción virtual, como por ejemplo MeshLab, Blender, Strata Desing 3D o Maya. Para el caso del presente artículo, se empleó la plataforma MeshLab ver. 1.3. 
Además, en el proceso de obtención del modelo 3D se generaron estimaciones de tres variables continuas de volumen (Figura 3 ) inferidas automáticamente en el ambiente AutoCAD 2013 (ver. 19.0) por medio del comando MASSPOP y siguiendo la propuesta de Sopena Vicién (2006), quien define:

» Masa vacío (Mv): masa del recipiente vacío;

» Masa bruto $(\mathrm{Mb})$ : masa total del recipiente y su contenido;

》Volumen bruto $(\mathrm{Vb})$ : capacidad o cantidad máxima de un recipiente.

Para obtener un control del rango o intervalo de error en los valores de $\mathrm{Vb}$ en el protocolo de reconstrucción 3D se exploró el valor del cálculo de volúmenes entre el cálculo directo de la única pieza cerámica completa del conjunto estudiado (sitio San Pablo, Figura 2) y la estimación efectuada a partir de los programas mencionados. De esta prueba resultó una ligera divergencia (2 y 1,88 litros respectivamente, Tabla 2).

Asimismo, por medio de los valores obtenidos de Mb se exploró la clasificación propuesta por Sopena Vicién (2006) sobre la movilidad o transportabilidad de las piezas. Para ello, se obtuvo el rango de la variable y se dividió en tres segmentos proporcionales que derivaron en tres categorías: dinámica ( 0,26 a 4,20 litros), estática-dinámica $(4,20$ a 8,14 litros) y estática (8,14 a 12,07 litros).

\section{Discusión de los resultados obtenidos}

La reconstrucción y visualización en 3D de las piezas reveló la variabilidad de las formas, de los tamaños y también de las capacidades o volúmenes del conjunto analizado (Figura 4). La facilidad del uso de herramientas informáticas permite que diversos investigadores hagan uso de esta herramienta y puedan potencializar el análisis cerámico. Respecto de las formas, hay que mencionar que dos piezas, LA2 (1) y EP2 (14), debieron tener originalmente al menos un asa lateral (Schuster, 2014), aunque estos segmentos no pudieron incorporarse en la reconstrucción virtual debido a que no se logró establecer la ubicación y el emplazamiento original de las mismas en las tareas de remontaje. Otro aspecto para aclarar es que en los casos de segmentos faltantes en los perfiles remontados, estos debieron ser proyectados o prolongados a partir de una línea de puntos para completar su respectiva reconstrucción en 2 y $3 \mathrm{D}$ (Figura 4). La prolongación de los perfiles de los cuerpos fue generada a partir del uso de formas geométricas básicas (por ejemplo elipsoide, cilindro, etc.), teniendo en cuenta además si las piezas a representar eran de contornos simples o compuestos. Para el caso de las bases, se consideró también que en la región se ha documentado una tendencia generalizada a bases cóncavas, ya sea en los materiales recuperados con técnicas arqueológicas (Schuster, 2014, 2016) como en las colecciones de museos regionales (Schuster, 2012, 2016).

Con relación a las capacidades o volúmenes brutos $(\mathrm{Vb})$, las reconstrucciones obtenidas dan cuenta de contenedores menores a un 1 litro $(n=5)$, de entre 1 y 7 litros $(n=7)$ y un único caso que supera los 10 litros (Tabla 2 y Figura 4). Concretamente, la media de $\mathrm{Vb}$ es de 3,2 litros, con un rango entre 0,2 y 10,9 litros. También, a partir de los cálculos estimados de masa bruta (o Mb), se obtuvo el peso total que habría tenido un contenedor completamente cargado según la estimación máxima de los volúmenes o su $\mathrm{Vb}$ (Tabla 2). Sin embargo, hay que tener en cuenta que estos rangos son el máximo considerado debido a que en las actividades de uso o durante el traslado de las piezas este debería haber sido menor, ya que es impracticable cocinar o trasladar alimentos o bebidas al borde de un recipiente. Sopena Vicién (2006) denomina a este valor como 


\begin{tabular}{|c|c|c|c|c|c|}
\hline Sitio & Piezas cerámicas & $\begin{array}{c}\text { Volumen bruto } \\
(\mathrm{Vb})^{*}\end{array}$ & $\begin{array}{c}\text { Masa vacío } \\
(\mathrm{Mv})^{\star *}\end{array}$ & $\begin{array}{l}\text { Masa bruto } \\
(\mathrm{Mb})^{*}\end{array}$ & $\begin{array}{l}\text { Capacidad } \\
\text { (Mb) }\end{array}$ \\
\hline San Román 1 & $\mathrm{PSR}_{1}(1)$ & 1,89 & 0,50 & 2,39 & Dinámica \\
\hline Los Abanicos 3 & $\operatorname{LABAN}_{3}(2)$ & 2,57 & 0,30 & 2,87 & Dinámica \\
\hline \multirow{3}{*}{ El Progreso 2} & $\mathrm{EP}_{2}(1)$ & 7,49 & 1,46 & 8,95 & Estática \\
\hline & $\mathrm{EP} 2(7)$ & 0,37 & 0,07 & 0,44 & Dinámica \\
\hline & $\mathrm{EP}_{2}$ (14) & 5,76 & 0,64 & 6,40 & Estática-Dinámica \\
\hline Las Ollas 1 & $\mathrm{LO}_{1}(14)$ & 1,34 & 0,23 & 1,57 & Dinámica \\
\hline San Pablo & $\mathrm{SP}(1)$ & 1,88 & 0,28 & 2,15 & Estática-Dinámica \\
\hline \multirow{2}{*}{ La Azucena 2} & $\mathrm{LA} 2(1)$ & 6,72 & 0,80 & 7,51 & Estática-Dinámica \\
\hline & $\mathrm{LA} 2(2)$ & 10,87 & 1,20 & 12,07 & Estática \\
\hline R. Elizalde 3 & $\mathrm{RE}_{3}$ (3) & 0,41 & 0,12 & 0,53 & Dinámica \\
\hline Cerro Avanzado & $\mathrm{CA}(4)$ & 0,74 & 0,02 & 0,76 & Dinámica \\
\hline Bahía Cracker 5 & $\mathrm{BC}_{5}(1)$ & 0,21 & 0,05 & 0,26 & Dinámica \\
\hline Bajo de los Huesos & $\mathrm{BH}(1)$ & 0,57 & 0,18 & 0,75 & Dinámica \\
\hline
\end{tabular}

Tabla 2. Datos métricos de las piezas reconstruidas en 3D y clasificación según la capacidad de la Mb sensu Sopena Vicién (2006). Referencias: "litros, $*$ kilos.

volumen neto $(\mathrm{Vn})$ y lo define como la capacidad máxima probable que las piezas podrían contener. Dado que esto implica determinar a priori cuál podría haber sido este límite máximo, en este trabajo se decidió no efectuar dicha medición. Al respecto, se tomó el dato de masa bruta, pero considerando que habría sido un volumen máximo no alcanzado en actividades habituales de uso.

Se estimó asimismo la masa vacío $(\mathrm{Mv})$ o el peso del recipiente vacío, obteniéndose valores que oscilan entre 0,02 y 1,46 kilos, de los cuales solo dos casos superan el kilo: la pieza LA2 (1), para la cual se determinó el mayor volumen de todo el conjunto (10,86 litros) y la vasija EP2 (1), que presentó una capacidad estimada en 7,49 litros (Tabla 2). El caso de estos contenedores pesados, pero que tienen diferente capacidad $o$ volumen, es un aspecto que se relaciona directamente con el espesor diferencial de las paredes (Tabla 1).

Complementariamente, los valores considerados para estimar el desplazamiento o movilidad probable de las piezas (obtenidos a través de la variable $\mathrm{Mb}$ ) muestran que la mayoría de las piezas de este conjunto se podrían clasificar como dinámicas $(\mathrm{n}=8)$, mientras que las restantes son estáticas-dinámicas $(\mathrm{n}=3)$ y estáticas $(\mathrm{n}=2)($ Tabla 2$)$.

\section{Consideraciones finales y perspectivas futuras}

$\mathrm{Al}$ utilizar programas de diseño digital se logró convertir el dibujo tradicional 2D en sólidos de revolución que posibilitaron obtener la morfología completa de las piezas parcialmente remontadas en el área de estudio. Sin embargo, cabe señalar que los contenedores elaborados sin torno, como los aquí considerados, no tienen una simetría perfecta, por lo que las imágenes obtenidas deben ser tomadas como modelos inferenciales y no como una representación real. No obstante, a partir de los modelos 3D obtenidos se realizaron los cálculos de volumen de las cerámicas reconstruidas que permitieron determinar que el conjunto analizado está representado por recipientes cuya capacidad varía entre 0,21 y 10,87 litros, con una media de 3,2 litros. 


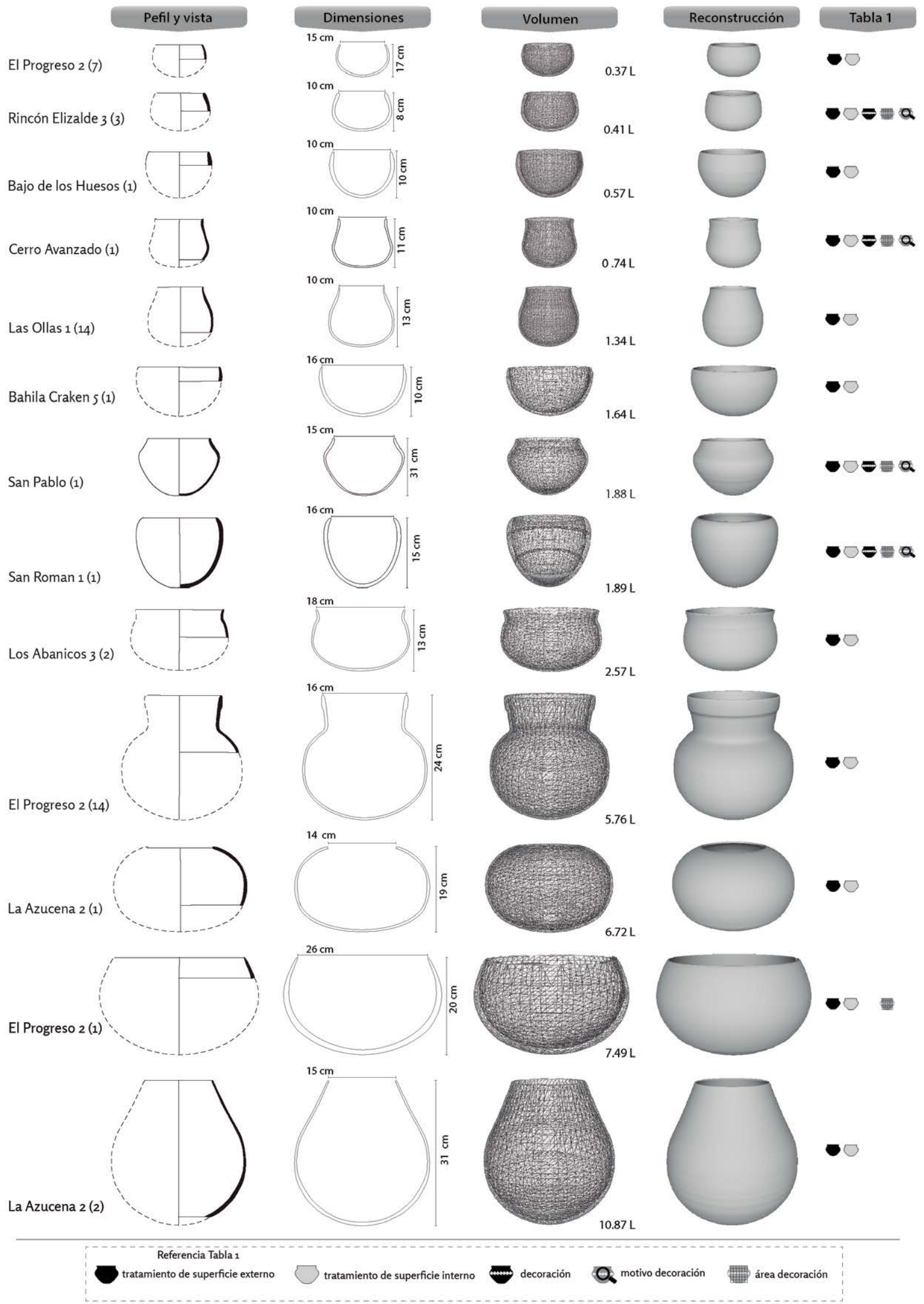

Figura 4. Perfil, dimensiones, volumen y reconstrucción 3D de las piezas cerámicas parcialmente remontadas en el área de estudio. Se señalan además los casos decorados haciendo referencia a los datos expuestos en la Tabla 1. 
La relación entre el tamaño y el volumen permite plantear aspectos relacionados con el desplazamiento o movilidad de las cerámicas durante las distintas tareas o actividades de uso en el pasado. También, el peso de los contenedores vacíos es un dato que se puede emplear para explorar la transportabilidad de las piezas en sociedades cazadorasrecolectoras móviles, como las aquí consideradas. Tomándose en cuenta la propuesta de Sopena Vicien (2006), se considera que la mayor parte del conjunto presentado está integrado por contenedores altamente dinámicos debido a que su peso y volumen se encuentran por debajo de los 0,50 kilos y los 2,57 litros, respectivamente. Los resultados obtenidos a partir de las reconstrucciones $3 \mathrm{D}$ avalan las interpretaciones alcanzadas anteriormente (Schuster, 2014), que postulan que estos grupos humanos habrían desarrollado una tecnología cerámica versátil y dinámica acorde a su estilo de vida, esto significa piezas que por su morfología y tamaño habrían sido multifuncionales, y que por su escaso peso, tenderían a ser livianas y fácilmente transportables.

Finalmente, cabe destacar que los datos expuestos aquí sobre el volumen y el peso del conjunto cerámico de la costa noreste del Chubut son los primeros que se tienen para esta tecnología en toda la Patagonia argentina bajo esta metodología de trabajo. Se espera que este tema, así como otras líneas de investigación, sean profundizados conforme se continúen implementando nuevas estrategias para el estudio de la cerámica de los grupos cazadores-recolectores de la región.

\section{Agradecimientos}

A Juan Ignacio Mihalich de Puerto Madryn, quién facilitó para su estudio la única pieza completa; a Roberto "Bobby" Taylor por las tareas de ensamblaje de fragmentos; a los arquitectos Viridiana Martínez y Adrián Flores (México) por la asesoría técnica CAD y al CONICET por la beca postdoctoral y doctoral latinoamericana otorgadas a los autores. Los materiales cerámicos aquí presentados fueron recuperados por la Dra. Julieta Gómez Otero (IDEAus, CENPAT-CONICET) en diversos trabajos de campo subsidiados por el CONICET y la National Geographic Society. 


\section{Q Referencias bibliográficas}

» Aldazabal, V. y Eugenio, E. (2009). Entre el fuego y el juego. La cerámica del sitio Rincón Chico 2/87. En E. Crivelli Montero, M. Fernández y M. Ramos (Comps.), Arqueología de Rescate en Rincón Chico, Provincia del Neuquén (pp. 163-186). Buenos Aires: Dunken.

» Arrigoni, G. (2002). Los ceramistas prehistóricos del valle del río Desaguadero, Parque Nacional Los Alerces, provincia del Chubut. Relaciones de la Sociedad Argentina de Antropología, XXVII, 395-412.

»Ávido, D. y Vitores, E. (2016). Aplicación de herramientas digitales para la reconstrucción tridimensional de soportes rupestres en el Noroeste Patagónico. En F. Oliva, A. Rocchietti y F. Solomita Banfi (Eds.), Imágenes Rupestres: Lugares y Regiones (pp. 403-414). Rosario: Universidad Nacional de Rosario.

»Carosio, S., Aguilar, J. P. y Bárcena, R. (2013). Reconstrucción 3D y calculo volumétrico de recipientes cerámicos. Alcances y limitaciones para el estudio y conservación cerámica de la tambería de Guancadol (provincia de La Rioja). La Zaranda de Ideas. Revista de Jóvenes Investigadores en Arqueología, 9(2), 57-76.

»Caviglia S., Borerro, L., Casiraghi, M., García, L. y Horwitz, V. (1982). Nuevos Sitios Arqueológicos para la Región de Bahía Solano (Chubut). Trabajo presentado en el VII Congreso Nacional de Arqueología Argentina. San Luis, Argentina.

» Ghiani Echenique, N. y León, P. (2013). Metodología 3D para la Reconstrucción de Formas Cerámicas en Contextos de Cazadores-recolectores. Sitio Las Marías (Partido de Magdalena, Provincia de Buenos Aires). Trabajo presentado en las IX Jornadas de Jóvenes Investigadores en Ciencias Antropológicas. Buenos Aires, Argentina.

» Ghiani Echenique N., Sokol, O. y Lozano, M. (2017). Reconstrucción virtual. Un aporte a la arqueología en tres dimensiones. Cuadernos del Instituto Nacional de Antropología y Pensamiento Latinoamericano, Series Especiales, 4(4), 20-29.

» Gómez Otero, J., Banegas, A., Caruso Fermé, L., Goye, M. S., Schuster, V., Svodoba, A. y Weiler, N. (2017b). Los primeros pobladores humanos: arqueología de la Bajada Colombo. En D. Udrizar Sauthier, G. Pazos y A. M. Arias (Eds.), San Pablo de Valdés. 10 años (pp. 228-247). Buenos Aires: Fundación Vida Silvestre y CONICET.

" Gómez Otero, J., Schuster, V. y Banegas, A. (2017a). Archaeology of Península Valdés: spatial and temporal variability in the human use of the landscape and geological resources. En P. Bouza y A. Bilmes (Eds.), Late Cenozoic of Península Valdés: an Interdisciplinary Approach (pp. 233-261). Londres: Springer.

»Gómez Otero, J., Schuster, V. y Constenla, D. (2014). Isótopos estables de carbono y nitrógeno y cromatografía gaseosa en cerámica del nordeste de la Provincia del Chubut (Patagonia Argentina). Arqueología, 20(2), 263-284.

" Gómez Otero, J, Schuster, V. y Svodoba, A. (2014). Fish and plants: the "hidden" resources in the archaeological record of the north-central Patagonian coast (Argentina). Quaternary International, 373, 72-81.

"Gradin, C. (1980). Secuencias radiocarbónicas del sur de la Patagonia argentina. Relaciones de la Sociedad Argentina de Antropología, XIV, 177-194.

"Irujo Ruiz, D. y Prieto Martínez, M. P. (2005). Aplicaciones 3D en cerámica prehistórica de contextos arqueológicos gallegos: un estudio sobre percepción visual. Arqueoweb, $7(2)$. 
» Rice, P. (1987). Pottery Analysis. A Sourcebook. Chicago: University of Chicago Press.

"Schuster, V. (2009). Petrografía de la cerámica arqueológica del nordeste del Chubut (Patagonia argentina). Primeros resultados. En S. Bertolino, R. Cattanéo y A. Izeta (Eds.), La Arqueometría en Argentina y Latinoamérica (pp. 103-108). Córdoba: Facultad de Filosofía y Humanidades, Universidad Nacional de Córdoba.

"Schuster, V. (2012). Organización de la tecnología cerámica en grupos cazadores recolectores de la costa centro-septentrional de Patagonia (Chubut, Argentina). (Tesis Doctoral inédita), Universidad del Centro de la Provincia de Buenos Aires, Argentina.

" Schuster, V. (2014). La organización tecnológica de la cerámica de cazadoresrecolectores. Costa norte de la provincia del Chubut (Patagonia argentina). Relaciones de la Sociedad Argentina de Antropología, XXXIX(1), 353-366.

»Schuster, V. (2015). Cerámica arqueológica de la costa, valle y meseta de la provincia del Chubut (Patagonia argentina): estudio comparativo preliminar de la composición de las pastas a través de la petrografía. Intersecciones en Antropología, 16(2), 353-366.

» Schuster, V. (2016). Estudios macro y microscópicos en la cerámica de cazadoresrecolectores de la costa oeste del golfo San Matías (prov. Río Negro, Argentina). En F. Mena (Ed.), Arqueología de Patagonia. De mar a mar (pp. 332-341). Buenos Aires: Ñire Negro.

"Schuster, V. y Banegas, A. (2010). Rayos X en la cerámica arqueológica de Patagonia: primeras experiencias para la costa y meseta central del Chubut. En J. Bárcena y $\mathrm{H}$. Chiavazza (Eds.), Arqueología Argentina en el Bicentenario de la Revolución de Mayo (Tomo V) (pp. 1987-1992). Mendoza: Universidad Nacional de Cuyo y CONICET.

» Schuster V., Banegas, A. y Taylor, R. (2013). Revelando imágenes... Rayos X en cerámicas arqueológicas y piezas experimentales. En A. Zangrando, R. Barberena, A. Gil, M. Giardina, L. Luna, C. Otaola, S. Paulides, L. Salgán y A. Tivoli (Eds.), Tendencias Teórico Metodológicas y Casos de Estudio en la Arqueología de Patagonia (pp. 233-242). San Rafael y Buenos Aires: Museo de Historia Natural de San Rafael, Sociedad Argentina de Antropología e Instituto Nacional de Antropología y Pensamiento Latinoamericano.

» Sopena Vicién, M. C. (2006). La investigación arqueológica a partir del dibujo informatizado de cerámica. Salduie 6. Estudios de Prehistoria y Arqueología, 6, 13-27. 